\title{
SOME FINITE IDENTITIES CONNECTED WITH POISSON'S SUMMATION FORMULA
}

\author{
by A. P. GUINAND
}

(Received 1st September 1959) .

\section{Introduction}

It is known that there is a connexion between the functional equation of the theta-function $\dagger$

$\theta_{3}(0 \mid \tau)=\sum_{n=-\infty}^{\infty} e^{\pi i n^{2} \tau}=(-i \tau)^{-\frac{1}{2}} \sum_{n=-\infty}^{\infty} e^{-\pi i n^{2} / \tau}=(-i \tau)^{-\frac{1}{2}} \theta_{3}\left(0 \mid-\frac{1}{\tau}\right)$

$$
\{I(\tau)>0\}
$$

and the reciprocity formula for Gauss sums. The latter formula is usually written as

$$
\sum_{n=0}^{q-1} e^{\pi i n^{2} p / q}=e^{\frac{1}{4} \pi i}\left(\frac{q}{p}\right)^{\frac{1}{2}} \sum_{n=0}^{p-1} e^{-\pi i n^{2} q / p}
$$

where $p$ and $q$ are positive integers, mutually prime, and not both odd. It can also be written as

$$
\sum_{n=-q}^{q \prime \prime} e^{\pi i n^{2} p / q}=e^{\frac{1}{4} \pi i}\left(\frac{q}{p}\right)^{\frac{1}{2}} \sum_{n=-p}^{p} e^{-\pi i n^{2} q / p},
$$

where the notation $\Sigma^{\prime \prime}$ is used to indicate that both the first and the last terms in the sums are to be halved, and the result (2) is true for all positive integers $\S$ $p$ and $q$.

The formula (1) can be deduced from Poisson's summation formula.\| The formula (2) can be derived from (1) by a method of Landsberg, $\uparrow$ or it can be deduced from Poisson's summation formula by a method of Dirichlet. $\dagger \dagger$ Thus we can describe (2) as a finite identity connected with the case (1) of Poisson's summation formula.

Now Poisson's summation formula can be written in the form $\sharp$

$$
\begin{aligned}
& \lim _{M \rightarrow \infty}\left\{\frac{1}{q} \sum_{n=1}^{M} f\left(\frac{n p}{q}\right)-\frac{1}{q} \int_{0}^{M} f\left(\frac{t p}{q}\right) d t\right\} \\
&=\lim _{N \rightarrow \infty}\left\{\frac{1}{\bar{p}_{n}} \sum_{n=1}^{N} g\left(\frac{n q}{p}\right)-\frac{1}{p} \int_{0}^{N} g\left(\frac{t q}{p}\right) d t\right\}
\end{aligned}
$$

† Cf. E. T. Whittaker and G. N. Watson, Modern Analysis (Cambridge, 1927), 468-469.

$\ddagger$ Sometimes called the formula of Genocchi and Schaar. See E. Lindelöf, Calcul des Résidus (Paris, 1905), 73-75 for references.

$\S$ It is trivially true if $p$ and $q$ are both odd, for then both sums vanish.

|| E. C. Titchmarsh, Fourier Integrals (Oxford, 1948), 60-64.

II M. Landsberg, J. für die reine und angewandte Math., 111 (1893), 234-253.

†† Cf. P. Bachmann, Die analytische Zahlentheorie (Leipzig, 1894), 156-161.

括 For conditions, see E. C. Titchmarsh, loc. cit., or A. P. Guinand, Annals of Math., 42 (1941), 591-603.

E.M.S.-B 
where $p$ and $q$ are real and positive, and $f(x), g(x)$ are Fourier cosine transforms with respect to the kernel $2 \cos 2 \pi x$; that is

$$
g(x)=2 \int_{0}^{\infty} f(t) \cos 2 \pi x t d t
$$

and

$$
f(x)=2 \int_{0}^{\infty} g(t) \cos 2 \pi x t d t .
$$

In the present paper $I$ show that finite identities of the form

$$
\frac{1}{q} \sum_{n=1}^{N q} f\left(\frac{n p}{q}\right)-\frac{1}{q} \int_{0}^{N q} f\left(\frac{t p}{q}\right) d t=\frac{1}{p} \sum_{n=1}^{N p} g\left(\frac{n q}{p}\right)-\frac{1}{p} \int_{0}^{N p} g\left(\frac{t q}{p}\right) d t
$$

hold when $p, q, N$ are any positive integers and either $\dagger$

$$
f(x)=g(x)=\psi(1+x)-\log x
$$

or +

$$
\begin{aligned}
& f(x)=x^{-1}\left\{\sum_{1 \leqq n \leqq x}^{\prime} 1-x\right\}, \\
& g(x)=\gamma+\log x-\sum_{1 \leqq n \leqq x}^{\prime} \frac{1}{n} .
\end{aligned}
$$

Both of these pairs of functions are transforms with respect to the kernel $2 \cos 2 \pi x$.

I also note some extensions of these results in section 4, and I make some remarks on the nature of the results in section 5 .

2. Finite Identities involving $\psi(1+x)-\log x$

In earlier papers $\S \mathrm{I}$ showed that $\psi(1+x)-\log x$ is self-reciprocal for the transformation (4), and that the formula

$$
\begin{aligned}
\sum_{n=1}^{\infty}\{\psi(1+n z) & \left.-\log n z-\frac{1}{2 n z}\right\}+\frac{1}{2 z}(\gamma-\log 2 \pi z) \\
= & \frac{1}{z} \sum_{n=1}^{\infty}\left\{\psi\left(1+\frac{n}{z}\right)-\log \frac{n}{z}-\frac{z}{2 n}\right\}+\frac{1}{2}\left(\gamma-\log \frac{2 \pi}{z}\right)(|\arg z|<\pi)
\end{aligned}
$$

can be deduced from Poisson's summation formula in the form (3).

We now prove the following result:

$\dagger \psi(x)$ denotes $\Gamma^{\prime}(x) / \Gamma(x)$.

$\ddagger$ The notation $\underset{1 \leq n \leqq x}{\Sigma^{\prime}}$ is used to indicate that the term $n=x$, if it occurs, is to be halved. Euler's constant is denoted by $\gamma$.

§ A. P. Guinand, J. London Math. Soc., 22 (1947), 14-18, and Edin. Math. Notes, 38 (1952), 1-4. The methods of the latter paper can also be used to prove Theorem 1, but a simpler method is used here to prove both Theorems 1 and 2. 
Theorem 1. If $F(x)=\psi(1+x)-\log x$ and $p, q, N$ are any positive integers then

$\frac{1}{q} \sum_{n=1}^{N q} F\left(\frac{n p}{q}\right)-\frac{1}{q} \int_{0}^{N q} F\left(\frac{t p}{q}\right) d t=\frac{1}{p} \sum_{n=1}^{N p} F\left(\frac{n q}{p}\right)-\frac{1}{p} \int_{0}^{N p} F\left(\frac{t q}{p}\right) d t$

We use the following lemma.

Lemma. If $p, q, N$ are any positive integers then

$$
\frac{1}{q} \sum_{n=1}^{q} \psi\left(1+p x+\frac{n p}{q}\right)-\frac{1}{p} \sum_{n=1}^{N p} \psi\left(1+q x+\frac{n q}{p}\right)=\log \frac{p}{q}
$$

and

$$
\frac{1}{q} \sum_{n=1}^{N q} \psi\left(1+p x+\frac{n p}{q}\right)-\frac{1}{p} \sum_{n=1}^{N p} \psi\left(1+q x+\frac{n q}{p}\right)=N \log \frac{p}{q} \ldots
$$

for all $x$ except those values which make the arguments of any $\psi$-function involved equal to $0,-1,-2, \ldots$.

Since $\dagger$

$$
\psi(1+z)=-\gamma+\sum_{m=1}^{\infty}\left(\frac{1}{m}-\frac{1}{m+z}\right)
$$

we have

$$
\begin{aligned}
& \frac{1}{q} \sum_{n=1}^{q} \psi\left(1+p x+\frac{n p}{q}\right)-\frac{1}{p} \sum_{n=1}^{p} \psi\left(1+q x+\frac{n q}{p}\right) \\
& \quad=\frac{1}{q} \sum_{n=1}^{q} \sum_{m=1}^{\infty}\left(\frac{1}{m}-\frac{1}{\left.m+p x+\frac{n p}{q}\right)}\right)-\frac{1}{p} \sum_{m=1}^{p} \sum_{n=1}^{\infty}\left(\frac{1}{n}-\frac{1}{n+q x+\frac{m q}{p}}\right) \\
& =\lim _{M \rightarrow \infty}\left\{\sum_{n=1}^{q} \sum_{m=1}^{M p}\left(\frac{1}{m q}-\frac{1}{m q+n p+p q x}\right)\right. \\
& =\lim _{M \rightarrow \infty}\left\{\sum_{m=1}^{M p} \frac{1}{m}-\sum_{n=1}^{M q} \frac{1}{n}\right\} \\
& =\log \frac{p}{q},
\end{aligned}
$$

since $m p+n q$ runs through the same set of integers when $1 \leqq m \leqq M p, 1 \leqq n \leqq q$ and when $1 \leqq m \leqq p, 1 \leqq n \leqq M q$. This proves (8); (9) follows if we replace $p, q$, and $x$ by $N p, N q$ and $x / N$ respectively.

† E. T. Whittaker and G. N. Watson, loc. cir., 241. 
Now consider the expression

$$
\begin{aligned}
\frac{1}{q} \sum_{n=1}^{N q}\{\psi(1+p x & \left.\left.+\frac{n p}{q}\right)-\log \left(p x+\frac{n p}{q}\right)\right\} \\
& -\frac{1}{q} \int_{0}^{N q}\left\{\psi\left(1+p x+\frac{t p}{q}\right)-\log \left(p x+\frac{t p}{q}\right)\right\} d t \\
& -\frac{1}{p} \sum_{n=1}^{N p}\left\{\psi\left(1+q x+\frac{n q}{p}\right)-\log \left(q x+\frac{n q}{p}\right)\right\} \\
& +\frac{1}{p} \int_{0}^{N p}\left\{\psi\left(1+q x+\frac{t q}{p}\right)-\log \left(q x+\frac{t q}{p}\right)\right\} d t
\end{aligned}
$$

Evaluating the integrals and using the lemma, we find that (10) is equal to

$$
\begin{aligned}
N \log \frac{p}{q}-\frac{1}{q} \sum_{n=1}^{N q} & \log \left(p x+\frac{n p}{q}\right)+\frac{1}{p} \sum_{n=1}^{N p} \log \left(q x+\frac{n q}{p}\right) \\
& -\frac{1}{p} \log \frac{\Gamma(1+p x+N p)}{\Gamma(1+p x)}+\frac{1}{q} \log \frac{\Gamma(1+q x+N q)}{\Gamma(1+q x)} \\
& +(x+N) \log p(x+N)-x \log p x-N \\
& -(x+N) \log q(x+N)+x \log q x+N . \ldots \ldots \ldots \ldots
\end{aligned}
$$

Now

$$
\begin{aligned}
\sum_{n=1}^{N q} \log \left(p x+\frac{n p}{q}\right) & =N q \log \frac{p}{q}+\log \left\{\prod_{n=1}^{N q}(q x+n)\right\} \\
& =N q \log \frac{p}{q}+\log \frac{\Gamma(1+q x+N q)}{\Gamma(1+q x)}
\end{aligned}
$$

and similarly

$$
\sum_{n=1}^{N p} \log \left(q x+\frac{n q}{p}\right)=N p \log \frac{q}{p}+\log \frac{\Gamma(1+p x+N p)}{\Gamma(1+p x)}
$$

Substituting these results in (11) we find that all terms cancel. Hence (10) vanishes identically, and we have proved:

Theorem 2. $\dagger$ If $F(x)=\psi(1+x)-\log x$ and $p, q, N$ are any positive integers

$\dagger$ The formula (12) can also be derived from a formula of Schobloch

$$
{ }_{n=0}^{q} \bar{\Pi}^{1} \Gamma\left(p x+\frac{n p}{q}\right)=(2 \pi)^{\frac{1}{2}(q-p)}\left(\frac{p}{q}\right)^{p q x+\frac{1}{2}(p q-p-q) p} \bar{\Pi}_{n=0}^{1} \Gamma\left(q x+\frac{n q}{p}\right)
$$

by logarithmic differentiation. Cf. N. Nielsen, Handbuch der Gammafunktion (Leipzig, 1906), 198. 
then

$$
\begin{aligned}
\frac{1}{q} \sum_{n=1}^{N q} F\left(p x+\frac{n p}{q}\right)- & \frac{1}{q} \int_{0}^{N q} F\left(p x+\frac{t p}{q}\right) d t \\
& =\frac{1}{p} \sum_{n=1}^{N p} F\left(q x+\frac{n q}{p}\right)-\frac{1}{p} \int_{0}^{N p} F\left(q x+\frac{t q}{p}\right) d t
\end{aligned}
$$

for all $x$ except those values which make the argument of any F-function involved equal to $0,-1,-2, \ldots$.

Theorem 1 follows on putting $x=0$ in (12).

\section{The Finite Identity involving}

$$
x^{-1}\left\{\sum_{1 \leqq n \leqq x}^{\prime} 1-x\right\} \text {. }
$$

First, we must show that the functions (6) are Fourier cosine transforms with respect to the kernel $2 \cos 2 \pi x$. Consider

$$
\begin{aligned}
& 2 \int_{0}^{N}\left\{\gamma+\log t-\sum_{1 \leqq n \leqq t}^{\prime} \frac{1}{n}\right\} \cos 2 \pi x t d t \\
& =\frac{\gamma}{\pi x} \sin 2 \pi N x+2 \int_{0}^{N} \log t \cos 2 \pi x t d t \\
& =\frac{\gamma}{\pi x} \sin 2 \pi N x+\frac{1}{\pi x} \log N \sin 2 \pi N x-\frac{1}{\pi x} \int_{0}^{N} \sin 2 \pi x t \frac{d t}{t} \\
& =\frac{\sin 2 \pi N x}{\pi x}\left(\gamma+\log N-\sum_{n=1}^{N} \frac{1}{\pi x} \sum_{n=1}^{N-1}\left(1+\frac{1}{2}+\frac{1}{3}+\ldots+\frac{1}{n}\right)\{\sin 2 \pi(n+1) x-\sin 2 \pi n x\}\right. \\
& =
\end{aligned}
$$

Making $N$ tend to infinity, the expression in brackets tends to zero, and we have

$$
\begin{aligned}
2 \int_{0}^{\rightarrow \infty}\left\{\gamma+\log t-\sum_{1 \leqq n \leqq t}^{\prime} \frac{1}{n}\right\} \cos 2 \pi x t d t \\
=\frac{1}{\pi x} \sum_{n=1}^{\infty} \frac{\sin 2 \pi n x}{n}-\frac{1}{2 x} \\
=x^{-1}\left\{\sum_{1 \leqq n \leqq x}^{\prime} 1-x\right\} .
\end{aligned}
$$


Both of the functions (6) obviously belong to $L^{2}(0, \infty)$ and are of bounded variation in any closed finite interval excluding $x=0$. Hence $\dagger$ we have:

Theorem 3. If $x>0$ and

$$
\begin{aligned}
& f(x)=x^{-1}\left\{\sum_{1 \leqq n \leqq x}^{\prime} 1-x\right\}, \\
& g(x)=\gamma+\log x-\sum_{1 \leqq n \leqq x}^{\prime} \frac{1}{n},
\end{aligned}
$$

then

$$
g(x)=2 \int_{0}^{-\infty} f(t) \cos 2 \pi x t d t
$$

and

$$
f(x)=2 \int_{0}^{-\infty} g(t) \cos 2 \pi x t d t .
$$

Now with the notation of Theorem 3 and $p, q, N$ any positive integers we have $\ddagger$

$$
\begin{aligned}
& \frac{1}{q} \sum_{n=1}^{N q} f\left(\frac{n p}{q}\right)-\frac{1}{q} \int_{0}^{N q} f\left(\frac{t p}{q}\right) d t \\
& =\frac{1}{q} \sum_{n=1}^{N q} \frac{q}{n p}\left\{\sum_{1 \leqq m \leqq n p / q}^{\prime} 1-\frac{n p}{q}\right\}-\frac{1}{p} \int_{0}^{N p} f(x) d x \\
& =\frac{1}{p} \sum_{1 \leqq m q \leqq n p \leqq N p q} \frac{1}{n}-\frac{1}{q} \sum_{n=1}^{N q} 1-\frac{1}{p} \int_{0}^{N p} x^{-1}([x]-x) d x \\
& =\frac{1}{p} \sum_{1 \leqq m q \leqq n p \leqq N p q} \frac{1}{n}-\frac{1}{p} \sum_{n=1}^{N k-1} n \int_{n}^{n+1} \frac{d x}{x} \\
& =\frac{1}{p} \sum_{1 \leqq m q \leqq n p \leqq N p q} \frac{1}{n}+\frac{1}{p} \log (N p) !-N \log N p .
\end{aligned}
$$

Similarly

$$
\begin{aligned}
\frac{1}{p} \sum_{m=1}^{N p} g\left(\frac{m q}{p}\right)-\frac{1}{p} \int_{0}^{N p} g\left(\frac{t q}{p}\right) d t & =\frac{1}{p} \sum_{m=1}^{N p}\left\{\gamma+\log \frac{m q}{p}-\sum_{1 \leqq n \leqq m q / p}^{\prime} \frac{1}{n}\right\}-\frac{1}{q} \int_{0}^{N q} g(x) d x \\
= & N\left(\gamma+\log \frac{q}{p}\right)+\frac{1}{p} \log (N p) !
\end{aligned}
$$

$\dagger$ E. C. Titchmarsh, loc. cit., 83.

¥ The notation $\Sigma \Sigma^{\prime}$ is to be interpreted here as indicating that the terms for which $m q=n p$ are to be halved. 


$$
\begin{aligned}
&- \frac{1}{p} \sum_{1 \leqq n p \leqq m q \leqq N p q} \frac{1}{n}-N \gamma-N \log N q+N \\
&+\frac{1}{q} \sum_{n=1}^{N q-1}\left(1+\frac{1}{2}+\frac{1}{3}+\ldots+\frac{1}{n}\right) \int_{n}^{n+1} d x \\
&=-\frac{1}{p} \sum_{1 \leqq n p \leqq m q \leqq N p q} \frac{1}{n}+\frac{1}{p} \log (N p) !-N \log N p+N \sum_{n=1}^{N q} \frac{1}{n} \ldots
\end{aligned}
$$

Subtracting (14) from (13) we have

$$
\begin{aligned}
& \left\{\frac{1}{q} \sum_{n=1}^{N q} f\left(\frac{n p}{q}\right)-\frac{1}{q} \int_{0}^{N q} f\left(\frac{t p}{q}\right) d t\right\}-\left\{\frac{1}{p} \sum_{n=1}^{N p} g\left(\frac{n q}{p}\right)-\frac{1}{p} \int_{0}^{N p} g\left(\frac{t q}{p}\right) d t\right\} \\
& =\frac{1}{p}\left\{\sum_{1 \leqq m q \leqq n p \leqq N p q} \frac{\sum^{\prime}}{n}+\sum_{1 \leqq n \leqq m q \leqq N p q} \frac{1}{n}\right\}-N \sum_{n=1}^{N q} \frac{1}{n} \\
& =\frac{1}{p}\left\{\sum_{m=1}^{N p} 1\right\}\left\{\sum_{n=1}^{N q} \frac{1}{n}\right\}-N \sum_{n=1}^{N q} \frac{1}{n} \\
& =0 .
\end{aligned}
$$

That is, we have:

Theorem 4. If $f(x)$ and $g(x)$ are as in Theorem 3 then the finite identity (5) holds for all positive integers $p, q, N$.

\section{Extended Forms of Finite Identities}

There is known to be an extension of the reciprocity for Gauss sums which can be written

$$
\sum_{n=0}^{q-1} e^{\pi i n^{2} p / q} \cos \frac{\pi r n}{q}=e^{\frac{1}{4 \pi i-t \pi i r^{2} / p q}}\left(\frac{q}{p}\right)^{\frac{1}{2} p} \sum_{n=0}^{-1} e^{-\pi i n^{2} q / p} \cos \frac{\pi r n}{p}
$$

where $p, q, r$ are positive integers $(p, q)=1$, and $p q+r$ is even. This can also be written

$$
\begin{aligned}
& \sum_{n=-q}^{q \prime} \exp \left\{\frac{\pi i p}{q}\left(n+\frac{r}{2 p}\right)^{2}\right\} \\
& \quad=\exp \left\{\frac{1}{4} \pi i\left(1+\frac{r^{2}}{p q}\right)\right\}\left(\frac{q}{p}\right)^{\frac{1}{2}} \sum_{n=-p}^{p} \exp \left\{-\frac{\pi i q}{p}\left(n+\frac{r}{2 q}\right)^{2}\right\},
\end{aligned}
$$

and in this form it is valid for all positive integers $p, q$ and all integers $r$. If $r=2 \mathrm{kpq}$ where $k$ is an integer, then (15) gives

$$
\sum_{n=k q-q}^{k q+q} e^{\pi i n^{2} p / q}=e^{\frac{1}{2 \pi i}}\left(\frac{q}{p}\right)^{\frac{1}{2}} \sum_{n=k p-p}^{k p+p} e^{-\pi i n^{2} q / p}
$$

which is obviously equivalent to (2). 
Theorem 2 can be regarded as an extension of Theorem 1 analogous to the extension (15) of (2). If we put $x=M$, where $M$ is an integer, in Theorem 2 then (12) implies that

$$
\begin{aligned}
\frac{1}{q} \sum_{n=M q+1}^{(M+N) q} F\left(\frac{n p}{q}\right)- & \frac{1}{q} \int_{M q}^{(M+N) q} F\left(\frac{t p}{q}\right) d t \\
& =\frac{1}{p} \sum_{n=M p+1}^{(M+N) p} F\left(\frac{n q}{p}\right)-\frac{1}{p} \int_{M p}^{(M+N) p} F\left(\frac{t p}{q}\right) d t .
\end{aligned}
$$

This result can be deduced from Theorem 1 by taking two different values of $N$ and subtracting, but the extended form (12) in this case is valid for all values of the additional parameter $x$ except a discrete set.

In the case of the finite identity of Theorem 4 it follows by subtraction that for positive integers $p, q, M, N,(M<N)$

$$
\frac{1}{q} \sum_{n=M q+1}^{N q} f\left(\frac{n p}{q}\right)-\frac{1}{q} \int_{M q}^{N q} f\left(\frac{t p}{q}\right) d t=\frac{1}{p} \sum_{n=M_{p+1}}^{N p} g\left(\frac{n q}{p}\right)-\frac{1}{p} \int_{M p}^{N p} g\left(\frac{t q}{p}\right) d t
$$

but there does not appear to be an extension analogous to (15) or (12). However, there does exist another type of extension. The methods of section 3 can be used to prove the following result.

Theorem 5. If $R(s)>0$ then the functions

$$
f_{s}(x)=x^{-s-1}\left\{\sum_{1 \leqq n \leqq x}^{\prime} n^{s}-\frac{x^{s+1}}{s+1}\right\}
$$

and

$$
g_{s}(x)=x^{s}\left\{\sum_{n \geq x}^{\prime} n^{-s-1}-\frac{x^{-s}}{s}\right\}
$$

are Fourier cosine transforms with respect to the kernel $2 \cos 2 \pi x$, and for all positive integers $p, q, N$.

$$
\begin{aligned}
\left\{\frac{1}{q} \sum_{1 \leqq n \leqq N q}^{\prime} f_{s}\left(\frac{n p}{q}\right)-\frac{1}{q} \int_{0}^{N q} f_{s}\left(\frac{t p}{q}\right) d t\right\} \\
-\left\{\frac{1}{p} \sum_{1 \leqq n \leqq N p}^{\prime} g_{s}\left(\frac{n q}{p}\right)-\frac{1}{p} \int_{0}^{N p} g_{s}\left(\frac{t q}{p}\right) d t\right\} \\
=-N f_{s}(N p) g_{s}(N q)+\frac{1}{4 N p q} . \cdots \ldots . .
\end{aligned}
$$

In the case $s=1$ we have

$$
f_{1}(N p)=(N p)^{-2}\left\{\sum_{1 \leqq n \leqq N p}^{\prime} 1-\frac{1}{2} N^{2} p_{i}^{2}\right\}=0,
$$

so the right-hand side of (18) reduces to $(4 N p q)^{-1}$, but no such simple reduction occurs for any other $s$ in $R(s)>0$. 


\section{Remarks on the Nature of the Finite Identities}

In order that there should be a finite identity of the type discussed above associated with a case of Poisson's summation formula it is essential that the pair of Fourier transforms involved should, in some sense, be functions of the same type. This is certainly true in the cases considered. For a case of Poisson's summation formula such as

$$
\frac{1}{2 q}+\frac{1}{q} \sum_{n=1}^{\infty} e^{-2 \pi n p / q}=\frac{1}{2 \pi p}+\frac{1}{\pi} \sum_{n=1}^{\infty} \frac{p}{p^{2}+n^{2} q^{2}}
$$

we cannot expect a corresponding finite identity such as

$$
\frac{1}{2 q}+\frac{1}{q} \sum_{n=1}^{N q} e^{-2 \pi n p / q}=\frac{1}{2 \pi p}+\frac{1}{\pi} \sum_{n=1}^{N p} \frac{p}{p^{2}+n^{2} q^{2}}
$$

to hold because it would imply relationships between transcendental functions and algebraic functions of an impossible nature.

Consequently it seems unlikely that any satisfactory general theory of finite identities associated with Poisson's summation formula could be developed, though we can note individual examples as in this paper.

The following trivial result is the only other example of such a finite identity which I have found. If $I(z)>0$ then Poisson's summation formula gives

$$
\sum_{n=-\infty}^{\infty} \operatorname{sech} \pi n z=\frac{1}{z} \sum_{n=-\infty}^{\infty} \operatorname{sech} \pi n / z
$$

The finite identity

$$
\sum_{n=-q}^{q} \sec \frac{\pi n p}{q}=\frac{i q}{p} \sum_{n=-p}^{p} \sec \frac{\pi n q}{p}
$$

corresponds to (19) in much the same way that (2) corresponds to (1). The formula (20) is certainly true if $p$ and $q$ are both odd integers, for then both sides vanish identically.

UNIVERSITY OF ALBERTA

EDMONTON, Alberta

CANADA 